\title{
Underinsurance on a Portfolio of Property Exposures in an Inflationary Environment *
}

\author{
by Gerard M. Dickinson ** and Leigh A. Roberts ***
}

\section{Introduction}

Underinsurance is generally recognised as being a serious problem for property insurance companies, often resulting in adverse underwriting results. Underinsurance can be broadly defined as a situation where the insurance cover on an exposure is less than the value of the property in the event of total loss. In property insurance, where premiums are commonly set proportional to sums insured, premium rates charged can be the same for property exposures, within a given risk class, whether these exposures are underinsured or not. Because small claims are for the most part more frequent than large claims, underinsurance can lead to inadequate pricing. In an inflationary environment, there is a tendency for underinsurance to increase in extent and degree within a property portfolio.

With respect to commercial and industrial property insurances, it is common for the insurance policy to allow the insurance company to reduce partial losses by some proportion in the event of underinsurance ${ }^{1}$. Such a proportion tends to be that between the sum insured and the value of the property at the time of loss; sometimes this reduction is applied only when the sum insured falls below a specified percentage, such as $80 \%$, of the value of the insured property. ${ }^{2}$ This practice of reducing claim payments to policyholders with respect to partial losses when underinsurance exists is referred to in the insurance parlance as average. In this paper we shall assume that the scaling down factor when applying this principle of average in the event of underinsurance will be a ratio of the sum insured to the total value of the property at the

* Paper presented at the Seventh Seminar of the European Group of Risk and Insurance Economists, University of Nottingham, September 1980. London.

** Senior Lecturer in Insurance and Corporate Finance, City University Business School,

*** B.I.A. Research Fellow, City University Business School, London.

1 If the underinsured property is totally destroyed by an insured peril the insurance company's payment will usually be limited to the sum insured.

2 For the variations that exist in practice in the UK see R. L. Carter: Handbook of Insurance, Kluwer Publishing Co., 1980, Section 3.2. 
time of settling the claim ; for ease of exposition, no distinction is made between the reporting and the settlement of a claim.

It is clear that the application of average is intimately related to the question of pricing and equity among policyholders within a given risk class. We shall concentrate more on the effectiveness of the practice of applying average as an ex-post adjustment for inadequate pricing. It is clear that if an insurance company were to anticipate the degree of underinsurance caused by inflation or otherwise at the outset of the policy and include an adequate allowance for this in determining their pure premiums, then there would be no need to apply average, other than for reasons of equity. In practice the right to apply average to partial losses is not always exercised by the insurer and there is not always a clear cut distinction between building in a loading for an expected incidence of underinsurance and applying this ex-post adjustment for inadequate pricing.

For ease of exposition, we shall assume a rather simplified model. It will be a one period model and not dynamic; we shall concentrate on the claims payments by an insurance company on a portfolio of property exposures; we shall implicitly ignore expenses and underwriting profit loadings. It will be assumed that at the outset the property exposures are fully insured and only become underinsured over time due to to the impact of inflation.

\section{Differential effects of inflation on claim amounts}

Before looking at the more general case of a portfolio of property exposures, let us first consider an individual exposure. For example such an exposure might be a building which is insured against fire loss over a period of time. We represent the distribution of the amount $z$ that an insurance company would be required to pay in the event of a claim at time $t$ as $P(z \mid t)$. The corresponding density function, $d P(z \mid t) / d z$, denoted by $P^{\prime}(z \mid t)$, might be expected to exhibit one of the two following shapes.
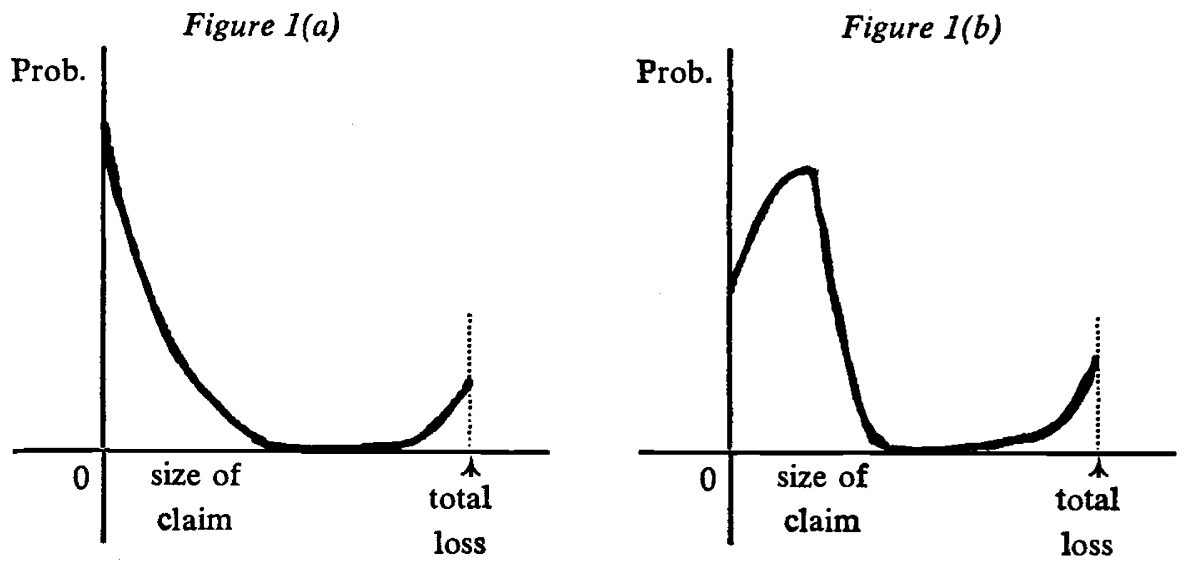

There is both empirical evidence and theoretical justification for assuming that such density functions would reveal decreasing probabilities as the size of claim increases 
over most of the range of possible claims. However, it would seem likely that the probabilities would tend to increase as the size of claim approached a total loss. ${ }^{3}$ With respect to a fire exposure, the point at which the probabilities begin to rise might be a point at which a fire would get increasingly out of control, therefore resulting in a total loss. But more generally with respect to property exposures, this could be expected to arise due to the comparatively high cost of repairs. With respect to very small claims, Figure 1b differs from Figure 1a in that it assumes that policyholders would be less disposed to make such claims either because the amounts involved would not warrant the administrative costs of doing so or because they might feel that small claims might adversely affect their future premium costs.

In the following, $z$ will denote the amount that an insurance company pays on a claim at time $t$, but if a claim occurs at exactly time $t=0$, we shall denote this by $y$. Hence $P(z \mid t)$ is the distribution function of a claim, conditional on there being a claim at time $t$, with the distribution function with respect to a claim at time $t=0$ being written as $P(y \mid 0)$. The distinction between $y$ and $z$ is made because we are concerned with the changing character of claims over time, so that $z$ can be written as some function of $y$.

A fundamental assumption in the ensuing analysis is that the claim distribution for a policy is changing over time only due to inflation. There is no intrinsic change in the propensity of the insured property to give rise to a claim. Another assumption is that if the insured property is damaged, it is repaired or replaced instantaneously and the policy continues in force. Later in the paper, when we consider a portfolio of policies, this latter assumption will not be important as long as the proportion of policies on which claims are made is small; but it is an essential assumption for one policy.

Formally, these two assumptions mean that :

$$
d P(y \mid 0)=d P(z \mid t)
$$

where the initial claim, $y$ at time 0 , has changed to $z$ at time $t$. This simply says that if a claim occurs at time $t$, the probability of that claim being an amount $z$ is the same as the probability of a claim at time 0 being an amount $y$.

Let us assume that the claim amount $y$ grows exponentially over time :

$$
z=y e^{\gamma t}
$$

$\gamma$ could be viewed as a constant, in which case the claims amounts would grow at a constant rate over time. It is more realistic to adopt a more general form such that $\gamma$ is a function of $y$. This permits us to allow for differential rates of inflation on claims of varying size. Indeed, it would be possible to specify $\gamma$ as also a function of time, viz :

3 Intuitively one would often expect the distribution of the claim amount to be discontinuous, with the amount claimed in the event of a total loss being an atom of the distribution and claims just below this maximum loss to have an extremely low or zero probability of occurrence. We approximate this distribution, for ease of exposition, by using a density function which increases smoothly but sharply from a very small value just before this point of maximum loss, as shown in Fig. 1. 


$$
z=y \exp \left[\int \gamma(y, \tau) d \tau\right]
$$

but this additional complication will not be considered.

At time 0 , the total value of the property exposure is scaled to unity. Since we are dealing with property exposures there will be a upper limit on the size of a claim at time 0 ; this would not necessarily be the case with respect to a liability exposure. The growth model assumed in the analysis here requires that an upper limit exists for it to be mathematically viable (see Appendix A).

For ease of exposition, $\gamma$ will be assumed to be simple linear function of $y$ :

$$
\gamma(y)=\gamma_{1}+\gamma_{2}(1-y) \quad \text { for } y \in[0,1]
$$

$\gamma_{1}$ is the rate at which the total value of the property is growing over this period, while $\gamma_{2}$ permits a differential rate of increase as the size of claim decreases. While $\gamma_{2}$ could in this general form be positive or negative, a positive value is more reasonable to assume in that partial losses are likely to grow at a faster rate than a total loss, due to the higher labour content with respect to smaller losses. While little published empirical data is available, there seems nevertheless to be some evidence to support the view that partial claims grow faster than total claims over time in respect of property insurance in an inflationary environment.

If a specific shape for the distribution of a claim with a density function similar in shape to that in Figure 1a is assumed, this permits some numerical values to be generated. More particularly, and for simplicity, let us assume that a time $t=0$ the density function has the following quadratic form:

$$
\begin{aligned}
a(y-b)^{2}+c, \quad \text { with } a, c>0 \\
1 / 2<b<1
\end{aligned}
$$

In general, the density function $P^{\prime}(z \mid t)$ is not quadratic for $t \neq 0$. If $\gamma_{2}>0$ the function will change shape broadly as shown in Figure 2, with a movement in the probability mass to the right, since smaller claim amounts are increasing more quickly than larger ones.

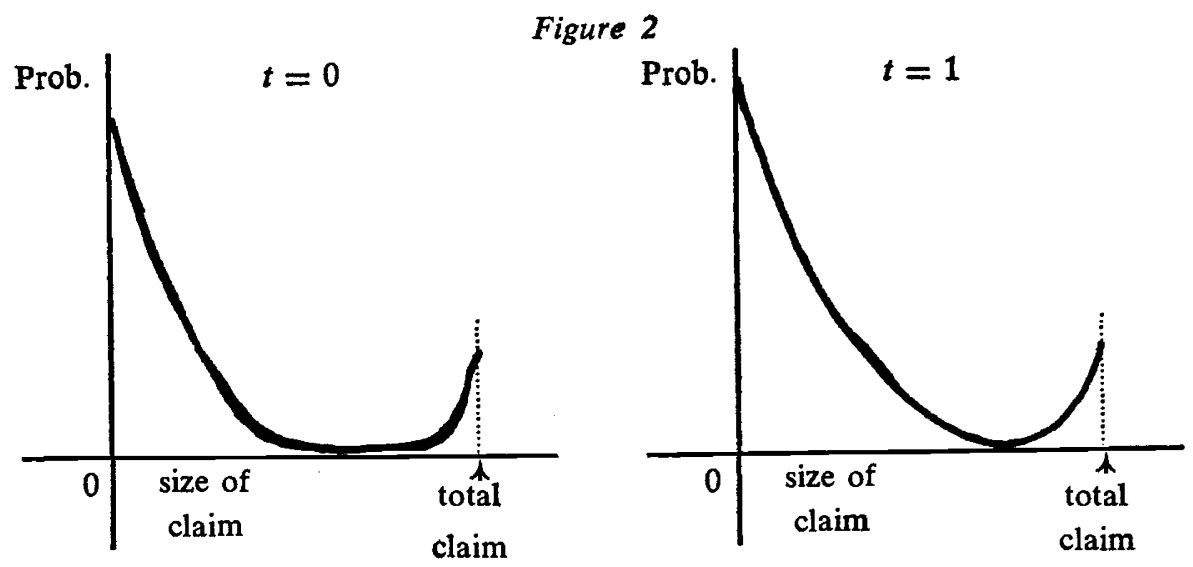


Appendix B indicates that the first two raw moments will increase if $\gamma_{2}$ increases. Let us consider the particular density function :

$$
8\left(y-\frac{2}{3}\right)^{2}+\frac{1}{9}
$$

The change in the moments over one period is shown below for different values of $\gamma_{1}$ and $\gamma_{2}$.

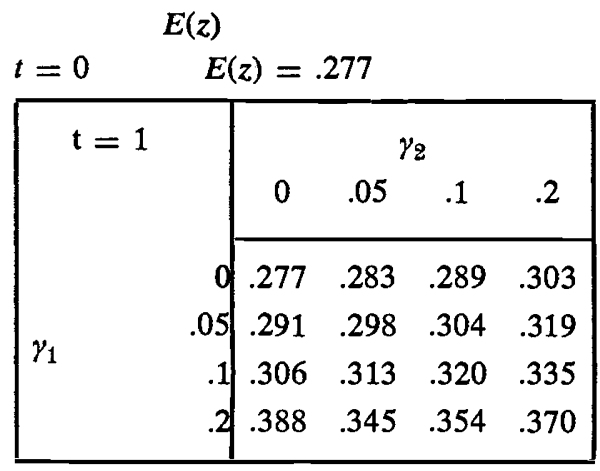

\begin{tabular}{|c|c|c|c|c|c|}
\hline \multicolumn{6}{|c|}{$E\left(z^{2}\right)$} \\
\hline$t=0$ & \multicolumn{5}{|c|}{$E\left(z^{2}\right)=.155$} \\
\hline \multirow[t]{2}{*}{$t=$} & & \multicolumn{4}{|c|}{$\gamma_{2}$} \\
\hline & & 0 & .05 & .1 & .2 \\
\hline \multirow{4}{*}{$\gamma_{1}$} & 0 & .155 & .159 & .163 & .172 \\
\hline & .05 & .171 & .175 & .180 & .190 \\
\hline & .1 & .189 & .194 & .199 & .210 \\
\hline & .2 & .231 & .237 & .243 & .256 \\
\hline
\end{tabular}

\section{Underinsurance, average and profit in a portfolio context}

Let us now look at a portfolio of $N$ policies on identical, independent exposures. We shall concentrate on the distribution of claims and for ease of exposition explicitly ignore expenses and underwriting profit loadings. The simple model advanced can be viewed as implying the following: 1) that the insurance company sets premiums to cover its pure premiums and expenses; no specific loading is included for underwriting profit ; 2) all premiums are paid at the beginning of the period and expenses are immediately incurred; thus the cash flow of premiums less expenses is available to earn interest prior to claims being paid ; 3 ) there is no allowance for interest made in determining pure premiums ; 4) all property exposures are considered to be fully insured at time 0 , but all will become underinsured due to the impact of inflation over the time period.

The particular issue that we shall focus attention on is the efficiency of the practice of average as an ex-post adjustment for inadequate pricing in the period of inflation. To put the analysis in a clearer perspective, let us consider two insurance companies, company $A$ and company $B$, that underwrite identical portfolios of property exposures as outlined above. Company $A$ is myopic and sets its pure premiums without regard for the impact of inflation on claims over time. It does, however, apply average and scales down claims when they occur, total and partial, by the ratio of the value of 
property at time 0 to the value of the property at time $t$. If the value of property at $t=0$ is one, then the scaling down factor in the event of a claim at time $t$ will be $e-\gamma_{1} t$.

On the other hand, company $B$ is forward-looking and has perfect foresight. It is aware of the inflation on claims over the period and builds these inflationary effects mto the pure premiums that it charges. It loads into premiums ex-ante the effects of inflation and hence underinsurance and pays claims in full without applying average ; indeed, it pays the full amount in the event of a total loss. Thus the pure premiums that it charges are equal to the expected monetary (undiscounted) value of the claim payments. In determming pure premiums, it is assumed that both companies have accurate estimates of the expected number of claims that will occur in their respective portfolios during the time period.

Let us now consider the distribution of claim payments on the portfolio of the $N$ identical, independent exposures. We shall consider the distribution of claims as discounted to time 0 . The discount rate $\delta$ is the rate of interest that the insurance company earns on the pre-payment of premiums, which we have implicitly assumed as the only source of profit from underwriting the portfolio. The moments of this distribution are derived in Appendix B. Here we shall only concern ourselves with the first moment and the particular case where the probability of an individual exposure suffering a claim is constant over the period.

The expected value of the discounted total claims paid on the portfolio by the insurance company over the period, assuming no average is applied, is :

$$
\begin{aligned}
\mu & =p_{1} \int_{0}^{1} \int_{0}^{e^{y_{1} t}} z e^{-\delta t} d P(z \mid t) d t \\
& +p_{2} \int_{0}^{1} \int_{0}^{1} \int_{0}^{y_{1} t_{2}} \int_{0}^{e^{\gamma_{1} t_{1}}}\left(z_{1} e^{-\delta t_{1}}+z_{2} e^{\left.-\delta t_{2}\right) d P\left(z_{1} \mid t_{1}\right) d P\left(z_{2} \mid t_{2}\right) d t_{1} d t_{2}}\right. \\
& +\ldots
\end{aligned}
$$

The first term $p_{1}$ is the probability that there will be exactly one claim on the total portfolio over the period; this one claim occurs at time $t$ with the probability $d t$, and the probability that the claim will be of size $z$ is $d P(z \mid t)$. In the second term, $p_{2}$ is the probability that there are exactly two claims over the year occurring at times $t_{1}$ and $t_{2}$. There are similar terms for three claims, four claims, etc.

In Appendix B, it is shown that this infinite series can be simplified to :

$$
\mu=E(n) \int_{0}^{1} \int_{0}^{e^{\gamma_{1} t}} z e^{-\delta t} d P(z \mid t) d t
$$

where

$$
E(n)=p_{1}+2 p_{2}+3 p_{3} \ldots .
$$

$E(n)$ represents the expected number of claims on the portfolio over the period. If the stronger assumption that the distribution of claims follows a Poisson process is made 
then $E(n)=N \lambda$, where $\lambda$ is the parameter for the Poisson process of claims on one policy and $N$ the number of policies. Hence transforming the above from variable $z$ to $y$ and deploying the simplifying assumption (2) we get :

$$
\mu=E(n) \int_{0}^{1} \int_{0}^{1} y e^{\left[\gamma_{1}+\gamma_{2}(1-y)\right] t} e^{-\delta t} d P(y \mid 0) d t .
$$

If the insurance company reduces claim payments due to underinsurance by applying average in the way discussed, this is equivalent to multiplying a claim at time $t$ by the factor $e^{-\gamma_{1} t}$. This gives

$$
\mu=E(n) \int_{0}^{1} \int_{0}^{1} y e^{\gamma_{2}(1-y) t} e^{-\delta t} d P(y \mid 0) d t .
$$

Let us now consider these two insurance companies, company $A$ and company $B$ under two scenarios : a) where high and low claims increase at the same rate of inflation; and b) where the claims increase at different rates of inflation. The pure premiums charged by company $A$ will be denoted as $P_{A}$ and the expected value of its discounted total claims payments as $\mu_{A}$. Similarly the pure premiums charged by company $B$ will be denoted by $\boldsymbol{P}_{B}$ and the expected value of its discounted total claim payments as $\mu_{B}$.

(a) Scenario $1: \gamma_{1}>0 ; \gamma_{2}=0$.

i) Consider first the case where $\delta=0$

$$
\begin{aligned}
& P_{A}=\mu_{A}=E(n) \iint y d P(y \mid 0) d t \\
& P_{B}=\mu_{B}=E(n) \iint y e^{\gamma_{1} t} d P(y \mid 0) d t .
\end{aligned}
$$

Here it can be seen that the application of average by company $A$ provides adequate compensation for the effects of inflation.

ii) Now consider the more realistic case where $\delta>0$ i.e. where a rate of interest can be earned on the pre-payment of premiums.

$$
\begin{aligned}
& P_{A}>\mu_{A}=E(n) \iint y e^{-\delta t} d P(y \mid 0) d t \\
& P_{B}>\mu_{B}=E(n) \iint y e^{\gamma_{1} t} e^{-\delta t} d P(y \mid 0) d t .
\end{aligned}
$$

Because claim payments are now discounted, a profit exists for both companies. But company $B$ will generate a higher profit than $A$, because it charges a higher pure premium thus enabling it to earn a higher level of investment income.

(b) Scenario 2: $\gamma_{1}>0 ; \gamma_{2} \neq 0$.

This is the more general case where claims are allowed to grow at different rates.

The more realistic case of $\gamma_{2}>0$ is assumed.

i) Again assume $\delta=0$.

$$
\begin{aligned}
& P_{A}<\mu_{A}=E(n) \iint y e^{\gamma_{2}(1-y) t} d P(y \mid 0) d t \\
& P_{B}=\mu_{B}=E(n) \iint y e^{\left[\gamma_{1}+\gamma_{2}(1-y)\right] t} d P(y \mid 0) d t .
\end{aligned}
$$


In this case company $A$ when applying average, viz reducing claims by $e^{-\gamma_{1} t}$, does not adjust for partial losses sufficiently. Hence company $A$ would incur a loss, with company $B$ again, by definition, breaking even.

ii) Again adopting the more realistic case $\delta>0$, we get

$$
\begin{aligned}
& P_{A} \lessgtr \mu_{A}=E(n) \iint y e^{\gamma_{2}(1-y) t} e^{-\delta t} d P(y \mid 0) d t \\
& P_{B}>\mu_{B}=E(n) \iint y e^{\left[\gamma_{1}+\gamma_{2}(1-y)\right] t} e^{-\delta t} d P(y \mid 0) d t .
\end{aligned}
$$

Again company $B$ will make a profit. It is difficult to say whether company $A$ will make a profit or not. This will depend on whether the rate of interest which it earns on the pre-payments of premiums is sufficient to offset the loss due to the inadequate downward scaling of partial claims. Even so it is clear that company $B$ will have a higher profit than company $A$ on two counts : a) it will have a higher investment income due to the higher level of its pure premiums and b) it will not suffer from the higher rate of increase on partial claims since it has included these in its premiums in advance.

\section{Downside risk}

It is also illuminating to analyse how the downside risk for companies $A$ and $B$ behave for differing values of $\gamma_{1}$ and $\gamma_{2}$. Downside risk is defined here as the probability that the discounted value of total claims exceeds the total pure premiums or, in other words, the probability that the company will incur a financial loss on underwriting the portfolio over the period. More specifically, we wish to calculate Prob $(x>P)$, where $x$ represents the discounted value of total claims and $P$ is the relevant total pure premiums.

It is not possible here to present a rigorous proof, but preliminary analysis strongly indicates that at least when $x$ has a normal distribution, then the downside risk for company $\boldsymbol{A}$ will always be greater than that for company $\boldsymbol{B}$, if either $\gamma_{1}$ or $\gamma_{2}$ is positive. To assume that the Central Limit Theorem applies such that the $x$ can be approximated by a normal distribution is appropriate for a large portfolio, given the earlier assumptions that the property exposures are independent and identical.

Moreover, the downside risk for Company $A$ can in general be expected to be greater when $\gamma_{2}>0$ than when $\gamma_{2}=0$, for a given $\gamma_{1}$.

\section{A numerical example}

A numerical example will help illustrate the general points raised in the paper so far. To simplify the exposition, the following further assumptions will be made :

(i) the period of analysis is 1 year ; 
(ii) at the beginning of the year, individual property exposures have a value of $£ 10,000$ and the density function of claims payments facing the insurance company for each exposure has the particular quadratic shape postulated in Figure 1 (a);

(iii) the portfolio contains 100,000 exposures and the expected number of claims during the year is 1000 ;

(iv) the annual rate of interest that can be earned on the pre-payment of premiums is $12 \%$ compounded continuously i.e. $\delta=.12$. Even though from an economic standpoint one would a priori expect $\delta$ and $\gamma$ to be correlated to some degree during the year, this is not allowed for specifically ;

(v) the discounted value of total claim payments is assumed to be normally distributed ; the two parameters needed to specify this distribution are calculated as outlined in Appendix $B$.

Let us now calculate and contrast the level of profit and the downside risk for company $\boldsymbol{A}$ and company $\boldsymbol{B}$ under the two scenarios for selected values of $\gamma_{1}$ and $\gamma_{2}$. It will be readily apparent that $P-\mu$ represents expected profits earned during the year discounted to the beginning of the year. It is more appropriate, therefore, to calculate the value $(P-\mu) e^{\delta}$ which is the profits valued at the end of the year. For convenience $(\boldsymbol{P}-\mu) e^{\delta}$ will be denoted by $\boldsymbol{V}$.

Scenario $1 \quad \gamma_{2}=0 ; \delta=.12$

Company $A$ :

\begin{tabular}{|l|c|c|c|c|}
\hline & \multicolumn{3}{|c|}{$\gamma_{1}$} \\
& 0 & .05 & .1 & .2 \\
\hline$V_{A}\left(f^{\prime} 000\right)$ & 181 & 181 & 181 & 181 \\
\hline Prob $\left(x_{A}>P_{A}\right)$ & .087 & .087 & .087 & .087 \\
\hline
\end{tabular}

Company $B$ :

\begin{tabular}{|l|c|c|c|c|}
\hline & \multicolumn{3}{|c|}{$\gamma_{1}$} \\
& 0 & .05 & .1 & .2 \\
\hline$V_{B}\left(\AA^{\prime} 000\right)$ & 181 & 187 & 193 & 206 \\
\hline Prob $\left(x_{B}>P_{B}\right)$ & .087 & .085 & .083 & .079 \\
\hline
\end{tabular}


It can be seen from the above that the profit level for company $A$ does not vary with changing values of $\gamma_{1}$. This is because (i) the pure premiums charged and hence the investment income generated is the same irrespective of the value of $\gamma_{1}$ and (ii) the application of average to claim payments exactly compensates for the inflationary increase in claims payments. On the other hand, for company $B$ the profit level increases as $\gamma_{1}$ increases, since a higher level of investment is produced by the additional loading in the pure premiums for the expected impact of inflation on claims.

The downside risk facing company $A$ does not change as $\gamma_{1}$ increases, but the downside risk facing company $B$ falls slowly as $\gamma_{1}$ increases. Thus except for the case where there is no inflation (i.e. $\gamma_{1} \leqslant 0$ ), company $A$ will have a lower level of profit and a higher downside risk than company $B$.

Scenario $2 \quad \gamma_{2} \geqslant 0 ; \delta=.12$

Company $A$ :

\begin{tabular}{|l|c|c|c|c|}
\hline & \multicolumn{3}{|c|}{$\gamma_{2}$} \\
& 0 & .05 & .1 & .2 \\
\hline$V_{A}\left(\AA^{\prime} 000\right)$ & 181 & 148 & 115 & 47 \\
\hline Prob $\left(x_{A}>P_{A}\right)$ & .087 & .133 & .195 & .366 \\
\hline
\end{tabular}

Company $B$ :

\begin{tabular}{|c|c|c|c|c|c|c|}
\hline & & & 0 & .05 & .1 & .2 \\
\hline & 0 & $\begin{array}{l}V_{B}\left(£^{\prime} 000\right) \\
\text { Prob }\left(x_{B}>P_{B}\right)\end{array}$ & $\begin{array}{l}181 \\
.087\end{array}$ & $\begin{array}{l}183 \\
.085\end{array}$ & $\begin{array}{l}186 \\
.083\end{array}$ & $\begin{array}{r}192 \\
.078\end{array}$ \\
\hline$\gamma_{1}$ & .05 & $\begin{array}{l}V_{B}\left(f^{\prime} 000\right) \\
\text { Prob }\left(x_{B}>P_{B}\right)\end{array}$ & $\begin{array}{l}187 \\
.085\end{array}$ & $\begin{array}{l}189 \\
.083\end{array}$ & $\begin{array}{l}192 \\
.081\end{array}$ & $\begin{array}{l}199 \\
.077\end{array}$ \\
\hline & .1 & $\begin{array}{l}V_{B}\left(£^{\prime} 000\right) \\
\text { Prob }\left(x_{B}>P_{B}\right)\end{array}$ & $\begin{array}{l}193 \\
.083\end{array}$ & $\begin{array}{l}196 \\
.081\end{array}$ & $\begin{array}{l}199 \\
.078\end{array}$ & $\begin{array}{l}205 \\
.076\end{array}$ \\
\hline & .2 & $\begin{array}{l}V_{B}\left(£^{\prime} 000\right) \\
\text { Prob }\left(x_{B}>P_{B}\right)\end{array}$ & $\begin{array}{l}206 \\
.079\end{array}$ & $\begin{array}{r}210 \\
.078\end{array}$ & $\begin{array}{l}213 \\
.076\end{array}$ & $\begin{array}{r}219 \\
.072\end{array}$ \\
\hline
\end{tabular}


As in scenario 1 , the level of profit and downside risk for company $A$ are independent of $\gamma_{1}$. But the numerical example illustrates how sensitive these financial characteristics are to increasing values of $\gamma_{2}$ for company $\boldsymbol{A}$. The sharp fall in profits and the increase in downside risk are directly traceable to the inadequacies of the averaging procedure as an ex-post pricing adjustment, when partial claims increase at a faster rate than total claims. In contrast, profits for company $B$ increase as either $\gamma_{1}$ or $\gamma_{2}$ increases due to the higher investment income produced by higher premiums ; moreover, while the downside risk does not change significantly, it does fall slowly with increasing values of either $\gamma_{1}$ or $\gamma_{2}$.

\section{Conclusion}

In this paper we have sought to look at the impact of inflation on property values over time and the related claims payments in the event of insured losses. Particular attention has been focused on the use of average as an ex-post pricing adjustment for underinsurance caused by inflation. A simple model has been developed which reveals the inadequacy of the conventional method of applying average when partial claims increase more quickly than total claims; that is, unless there is some initial loading in premiums to allow for this.

The reader will discern that this general point regarding the application of average could be made without the introduction of company $B$ as a point of contrast. Even so it is useful to introduce such a contrast, since it makes more explicit the interdependence between the ex-ante loading of premiums for inflation and the ex-post pricing adjustment of average. The question whether company $B$ could in a competitive market charge these higher rates than company $A$ and attract some share of the market is a subject for further analysis. Even without detailed analysis, it is possible to hypothesise that there would be conditions under which this could be so, since company $B$ is offering greater protection to policyholders than company $\boldsymbol{A}$. A key factor in such an analysis would clearly be the distribution of the degrees of risk aversion among policyholders in the market. The growth of index-linked policies in recent years, at higher premiums, is some empirical evidence of this. 4

Average is applied by insurance companies also to ensure some equity between policyholders within a portfolio. The issue of equity has not been addressed here directly, although the findings of the analysis indicate that the usual adjustment is unlikely to be adequate when partial claims grow at a faster rate than total claims. The analysis adopted earlier could be extended to develop this case, but we would need to permit property exposures to vary by size, for a particular risk class.

4 Company B can be considered to be a company issuing index-linked policies with perfect foreknowledge of $\gamma_{1}$ and $\gamma_{2}$. 
What issues of practical significance are suggested by the paper? Firstly, we stress the importance to an insurance company of investigating whether claim amounts can be expected to grow at different rates in an inflationary environment and, if so, of estimating these rates over time. Secondly, we observe that insurance companies should recognise more than they appear to do so the interdependence between building loadings ex-ante into pricing to allow for underinsurance caused by inflation or otherwise and applying average ex post to compensate for such underinsurance; this implies closer coordination between underwriting and claims departments. Thirdly, if average is applied in the interests of achieving equity between policyholders within a particular risk class, it may be more appropriate to devise and apply a sliding or variable scale of adjustment to allow for differing degrees of underinsurance, if partial claims are likely to continue to grow at significantly different rates from total claims.

It will be readily apparent that the simplified model adopted here can be extended. The portfolio of risk exposures considered could be permitted to vary in size and so could their propensity to have a claim over time. Specific allowance could be made in the model for expenses, underwriting profit and an initial stock of capital and free reserves ; moreover the simple rate of interest which the insurance company is deemed to earn can be linked to a portfolio of financial assets. It is the intention of the authors to develop the model further within a stochastic framework in order to explore the implications of different pricing policies, including the indexing of premiums and sums insured, on the rate of return on capital and the probability of ruin of an insurance company in the context of an inflationary environment.

\section{Appendix A}

Consider two different claim amounts $y$ and $y^{*}$ at time 0 , which increase exponentially over time at rates $\gamma(y)$ and $\gamma\left(y^{*}\right)$ respectively to amounts $z$ and $z^{*}$ at time $t$ :

$$
z=y e^{\gamma(y) t} ; \quad z^{*}=y^{*} e^{\gamma\left(y^{*}\right) t} .
$$

Suppose the smaller amount grows more quickly, specifically :

$$
y<y^{*} \text { and } \gamma(y)>\gamma\left(y^{*}\right)
$$

Let $T$ be the time when both initial amounts accumulate to the same value, which is given by

$\begin{array}{ll} & y e^{\gamma(y) T}=y^{*} e^{\gamma\left(y^{*}\right) T} \\ \text { Then } & z<z^{*} \text { for } t<T \\ \text { and } & z>z^{*} \text { for } t>T .\end{array}$

It is not possible to state unequivocally what probability to assign to a value of $z$ occurring at time $T$, since it could spring from either $y$ increasing at $\gamma(y)$, in which case the probability would be $d P(y \mid 0)$ or from $y^{*}$ increasing at $\gamma\left(y^{*}\right)$, in which case the probability would be $d P\left(y^{*} \mid 0\right)$. 
Thus unless $\gamma(y)$ is a non-decreasing function of $y$, there is some time limit to the validity of this simple growth model : the time when the two different initial amounts catch up to each other. Before this point in time it is possible to associate a claim $z$ at time $t$ with a unique pre-image $y$ at time 0 .

Consider again,

$$
z=y e^{\gamma(y) t}
$$

Differentiating :

$$
z^{\prime}(y)=e^{\gamma(y) t}\left[1+y t \gamma^{\prime}(y)\right]
$$

Relation (3) is invertible i.e. defines $y$ as a function of $z$, provided $z^{\prime}(y) \neq 0$. Adopting the linear form for $\gamma(y)$ :

$$
\begin{array}{ll} 
& \gamma(y)=\gamma_{1}+\gamma_{2}(1-y), \\
\text { we see that } & z^{\prime}(y)=0 \quad \text { if } \\
\text { (4) } & 1-y t \gamma_{2}=0 .
\end{array}
$$

If $\gamma_{2}<0$, this can never be satisfied; while if $\gamma_{2}>0$, the model breaks down (in the sense that a particular $z$ need not correspond to a unique $y$ ) at the first time $t$ such that

$$
t=\frac{1}{\gamma_{2} y}
$$

for some $y$. If $y$ has no upper bound (5) shows that the model is not valid for any time period; hence it is necessary to assume an upper limit on possible claims $y$ at time 0 . Choosing a monetary unit such that this upper limit is one, the model remains valid as long as

$$
t<\frac{1}{\gamma_{2}}
$$

For instance, if the smallest claims increase at $10 \%$ more than the highest claim, i.e. $\gamma_{2}=.1$, the model breaks down after 10 periods.

This can be further elucidated as follows. Consider initial values $y$ and $y-\varepsilon$, with $\varepsilon$ an infinitesimally small amount. These accumulate to the same value at time $t$, where

$$
y e^{\gamma(y) t}=(y-\varepsilon) e^{\gamma(y-\varepsilon) t} .
$$

That is,

$$
\begin{aligned}
& y e^{\gamma_{1} t} e^{\gamma_{2}}(1-y) t=(y-\varepsilon) e^{\gamma_{1} t} e^{\gamma_{2}(1-y+\varepsilon) t} \\
& \begin{aligned}
y & =(y-\varepsilon) e^{\gamma_{2}} \varepsilon t \\
& =(y-\varepsilon)\left(1+\gamma_{2} \varepsilon t+o(\varepsilon)\right) \\
& =y-\varepsilon\left(1-\gamma_{2} y t\right)+o(\varepsilon) .
\end{aligned}
\end{aligned}
$$


Thus the amount $y$ and the amount infinitesimally less than $y$ catch up to each other when

$$
1-\gamma_{2} y t=0
$$

which is (4) again. The break down of the model is apparent, since the pre-image at time 0 of

$$
y e^{r(y) t}
$$

can be either $y$ or $y-\varepsilon$.

The upper limit of 1 assumed for $y$ in the model means that the model is valid for up to 10 periods for $\gamma_{2}=.1$, up to 5 periods for $\gamma_{2}=.2$ etc.

This is not to say that the model is at all sensible over the whole period $\left[0, \frac{1}{\gamma_{2}}\right]$. Let $\gamma_{1}=\gamma_{2}=.1$, and consider the following accumulations :

\begin{tabular}{|c|c|c|c|c|}
\hline $\begin{array}{c}\text { Initial } \\
\text { amount }\end{array}$ & $\begin{array}{c}\text { Accumulated } \\
\text { amount after } \\
\text { 5 periods }\end{array}$ & $\begin{array}{c}\text { Percentage } \\
\text { of total }\end{array}$ & $\begin{array}{c}\text { Accumulated } \\
\text { amount after } \\
\text { 10 periods }\end{array}$ & $\begin{array}{c}\text { Percentage } \\
\text { of total }\end{array}$ \\
\hline 0 & 0 & 0 & 0 & 0 \\
.25 & .60 & 36 & 1.44 & 53 \\
.75 & 1.06 & 64 & 2.24 & 82 \\
1 & 1.40 & 85 & 2.62 & 96 \\
\hline
\end{tabular}

Since lower claims accumulate more quickly than higher claims, as time passes more and more of the claims have low pre-images. For instance, after 5 periods, the first $64 \%$ of claims (by size) arise from the lower half of the original claims at time 0 ; after 10 periods this has risen to $82 \%$. Note that $\gamma_{1}$ influences the accumulated amounts but the distribution is only distorted by $\gamma_{2}$, which, since it exceeds zero, is moving the mass of the distribution to the right. It is unlikely that we would believe in so much distortion even over a long period, and one must consider the useful life of the model to be less than the mathematically possible $\frac{1}{\gamma_{8}}$ periods for the simple linear case.

\section{Appendix $\mathbf{B}$}

In this appendix, the moments are derived for the total discounted value of the claims paid on a portfolio of policies covering $N$ identical, independent exposures. All integrals are assumed to exist. 
Let $p_{i}$ denote the probability of $i$ claims occurring over the period $[\tau, \tau+1)$, and $d Q(t)$ the probability that a claim occurs at time $t$, conditional on there being exactly one claim over $[\tau, \tau+1)$. Then the $k^{\text {th }}$ raw moment of the total claim payments (discounted to time 0 ) for the period $[\tau, \tau+1)$ is :

$$
\begin{aligned}
& a_{k \tau}=p_{1} \int_{\tau}^{\tau+1} \int_{0}^{e^{\gamma_{1} t}}\left(z e^{-\delta t}\right)^{k} d P(z \mid t) d Q(t)
\end{aligned}
$$

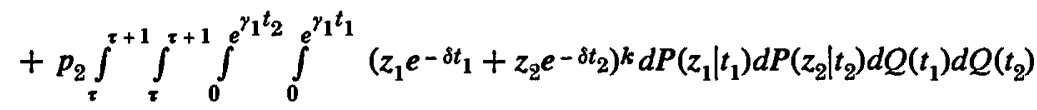

$$
\begin{aligned}
& +\ldots \text { etc. }
\end{aligned}
$$

Consider now $k=1$, the first part of the second term becomes :

$$
\begin{aligned}
& p_{2} \int_{\tau}^{\tau+1} \int_{\tau}^{\tau+1} \int_{0}^{e^{\gamma_{1} t_{2}}} \int_{0}^{\gamma_{1} t_{1}} z_{1} e^{-\delta t_{1} d P\left(z_{1} \mid t_{1}\right) d P\left(z_{2} \mid t_{2}\right) d Q\left(t_{1}\right) d Q\left(t_{2}\right)} \\
& =p_{2} \int_{\tau}^{\tau+1} \int_{0}^{\gamma^{\gamma_{1} t_{1}}} z_{1} e^{-\delta t_{1} d P\left(z_{1} \mid t_{1}\right) d Q\left(t_{1}\right) .}
\end{aligned}
$$

The second part of the second term reduces similarly, and so on for higher order integrals, such that

$$
a_{1 \tau}=\left(p_{1}+2 p_{2}+3 p_{3}+\ldots\right) \int_{\tau}^{\tau} \int_{0}^{e^{\gamma_{1}} t} z e^{-\delta t} d P(z \mid t) d Q(t)
$$

If $\boldsymbol{n}$ denotes the number of claims,

$$
a_{1 \tau}=E(n) \int_{\tau}^{\tau} \int_{0}^{e^{\gamma_{1} t}} z e^{-\delta t} d P(z \mid t) d Q(t)
$$

This is just what one would expect, since the double integral is the expected (discounted) payment on one claim.

For ease of exposition, let us define :

$$
\beta_{k \tau}=\int_{\tau}^{\tau+1} \int_{0}^{e^{\gamma_{1} t}}\left(z e^{-\delta t}\right)^{k} d P(z \mid t) d Q(t)
$$

such that

$$
\alpha_{1 \tau}=E(n) \beta_{1 \tau} .
$$


For $k=2$, (6) becomes

(8)

$$
\begin{aligned}
\alpha_{2 x} & =p_{1} \int_{\tau}^{t+1} \int_{0}^{e_{1} t}\left(z e^{-\delta t}\right)^{2} d P(z \mid t) d Q(t) \\
& +p_{2} \int_{\tau}^{t+1} \int_{\tau}^{t+1} \int_{0}^{\gamma_{1} t_{2}} \int_{0}^{\gamma_{1} t_{1}}\left(z_{1} e^{-\delta t_{1}}+z_{2} e^{-\delta t_{2}}\right)^{2} d P\left(z_{1} \mid t_{1}\right) d P\left(z_{2} \mid t_{2}\right) d Q\left(t_{1}\right) d Q\left(t_{2}\right) \\
& +\ldots \text { etc. }
\end{aligned}
$$

The $r^{\text {th }}$ term in (8) is

$$
p_{r}=\int_{i}^{\tau+1} \cdots \int_{0}^{e^{\gamma_{1} t_{1}}}\left(\sum z_{i} e^{-\delta t_{i}}\right)^{2} \Pi d P\left(z_{i} \mid t_{i}\right) \Pi d Q\left(t_{i}\right) .
$$

Each crossed term in the expansion of the square contributes the same value to $\alpha_{2 x}$ and so the $r^{\text {th }}$ term becomes

$$
p_{r}\left(r \beta_{2 x}+{ }^{r} \boldsymbol{P}_{2} \beta^{2}{ }_{1 \tau}\right)
$$

where

$$
{ }^{r} \boldsymbol{P}_{k}=r(r-1) \ldots(r-k+1)
$$

the number of permutations of $r$ objects taken $k$ at time.

Hence from (8)

$$
\begin{aligned}
\alpha_{2 v} & =p_{1} \beta_{2 v}+\sum_{r=2}^{\infty} p_{r}\left(r \beta_{2 \tau}+r P_{2} \beta^{2}{ }_{1 \tau}\right) \\
& =E(n) \beta_{2 \tau}+\beta^{2}{ }_{1 \tau} \sum_{r=2}^{\infty} r(r-1) p_{r} \\
& =E(n) \beta_{2 \tau}+\beta^{2}{ }_{1 \tau} \sum_{r=1}^{\infty} r(r-1) p_{r} \\
& =E(n) \beta_{2 \tau}+\beta^{2}{ }_{1 \tau}\left(E\left(n^{2}\right)-E(n)\right) .
\end{aligned}
$$

Proceeding similarly for the higher moments :

$$
\begin{aligned}
\alpha_{1 \tau} & =E(n) \beta_{1 \tau} \\
\alpha_{2 \tau} & =E(n) \beta_{2 \tau}+\beta^{2}{ }_{1 \tau} E\left({ }^{n} P_{2}\right) \\
\alpha_{3 x} & =E(n) \beta_{3 \tau}+3 \beta_{1 \tau} \beta_{2 \tau} E\left({ }^{n} P_{2}\right) \\
& +\beta^{3}{ }_{1 \tau} E\left({ }^{n} P_{3}\right)
\end{aligned}
$$


Consider the definition of $\beta_{k \tau}$ :

$$
\begin{aligned}
\beta_{k \tau} & =\int_{\tau}^{\tau+1} \int_{0}^{e^{\gamma_{1} t}}\left(z e^{-\delta t}\right)^{k} d P(z \mid t) d Q(t) \\
& =\int_{\tau}^{\tau+1} \int_{0}^{1}\left\{y e^{\left[\gamma_{1}+\gamma_{2}(1-y)\right] t} e^{-\delta t}\right\}^{k} d P(y \mid 0) d Q(t) .
\end{aligned}
$$

Since $(1-y)$ and $t$ are non-negative over the region of integration,

$$
e^{\gamma_{2}}(1-y) t
$$

increases with $\gamma_{2}$ over this region, and so $\beta_{k \tau}$ increases with $\gamma_{2}$ for all $k \geqslant 0$ and for all $\tau \geqslant 0$. Thus, from (9) it can be seen that the first three raw moments of the discounted payments for period $[\tau, \tau+1)$ increase with $\gamma_{2}$. This is the case for all the raw moments $a_{k \tau}$ for all non-negative $k$ and $\tau$.

Letting $x$ represent the discounted value of payments :

$$
\begin{array}{ll} 
& E\left(x^{2}\right)=a_{2 \tau} \\
\text { So } & \operatorname{Var}(x)=a_{2 \tau}-\alpha_{1 \tau}^{2} \text {. }
\end{array}
$$$$
E(x)=a_{1 \tau}
$$

In general then, it is not possible to state whether $\operatorname{Var}(x)$ increases with $\gamma_{2}$, but it is possible to do so when the distribution of claims over the year has the same expectation and variance; the Poisson distribution has this property.

If $n$ is now the number of claims in the year $[\tau, \tau+1)$,

$$
\operatorname{Var}(n)=E\left(n^{2}\right)-E(n)^{2}
$$

so that

$$
\begin{array}{ll}
E(n)=\operatorname{Var}(n) \\
<=> & E(n)=E\left(n^{2}\right)-E(n)^{2} \\
<=> & E\left(n^{2}\right)-E(n)=E(n)^{2} \\
<=> & a_{2 \tau}=E(n) \beta_{2 \tau}+\beta^{2}{ }_{1 \tau} E(n)^{2} \quad \text { from (9). }
\end{array}
$$

Now $\quad a_{1 \tau}=E(n) \beta_{1 \tau} \quad$ from (9) and

Hence $\quad \operatorname{Var}(x)=E(n) \beta_{2 \tau}$.

So if claims form a Poisson process in time, $\operatorname{Var}(x)$ increases with $\gamma_{2}$. Given that the parameter of Poisson process of claims for one policy is denoted by $\lambda$, the parameter of the Poisson process of claims on a portfolio of size $N$ is $N \lambda$. The first two moments of this process are :

$$
\begin{aligned}
& E(x)=N \lambda \beta_{1 \tau} \\
& \operatorname{Var}(x)=N \lambda \beta_{2 \tau} .
\end{aligned}
$$

